2013

\title{
Variability of the pulsed radio emission from the large magellanic cloud pulsar PSR J0529-6652
}

F. Crawford

D. Altemose

H. Li

D. R. Lorimer

Follow this and additional works at: https://researchrepository.wvu.edu/faculty_publications

\section{Digital Commons Citation}

Crawford, F.; Altemose, D.; Li, H.; and Lorimer, D. R., "Variability of the pulsed radio emission from the large magellanic cloud pulsar PSR J0529-6652" (2013). Faculty Scholarship. 427.

https://researchrepository.wvu.edu/faculty_publications/427 


\title{
VARIABILITY OF THE PULSED RADIO EMISSION FROM THE LARGE MAGELLANIC CLOUD PULSAR PSR J0529-6652
}

\author{
F. CrawFord ${ }^{1}$, D. Altemose ${ }^{1}$, H. LI $^{1}$, AND D. R. LORIMER ${ }^{2}$ \\ ${ }^{1}$ Department of Physics and Astronomy, Franklin and Marshall College, P.O. Box 3003, Lancaster, PA 17604, USA \\ ${ }^{2}$ Department of Physics, West Virginia University, Morgantown, WV 26506, USA \\ Received 2012 September 4; accepted 2012 November 17; published 2012 December 19
}

\begin{abstract}
We have studied the variability of PSR J0529-6652, a radio pulsar in the Large Magellanic Cloud (LMC), using observations conducted at $1390 \mathrm{MHz}$ with the Parkes $64 \mathrm{~m}$ telescope. PSR J0529-6652 is detectable as a single pulse emitter, with amplitudes that classify the pulses as giant pulses. This makes PSR J0529-6652 the second known giant pulse emitter in the LMC, after PSR B0540-69. The fraction of the emitted pulses detectable from PSR J0529-6652 at this frequency is roughly two orders of magnitude greater than it is for either PSR B0540-69 or the Crab pulsar (if the latter were located in the LMC). We have measured a pulse nulling fraction of $83.3 \% \pm 1.5 \%$ and an intrinsic modulation index of $4.07 \pm 0.29$ for PSR J0529-6652. The modulation index is significantly larger than values previously measured for typical radio pulsars but is comparable to values reported for members of several other neutron star classes. The large modulation index, giant pulses, and large nulling fraction suggest that this pulsar is phenomenologically more similar to these other, more variable sources, despite having spin and physical characteristics that are typical of the unrecycled radio pulsar population. The large modulation index also does not appear to be consistent with the small value predicted for this pulsar by a model of polar cap emission outlined by Gil \& Sendyk. This conclusion depends to some extent on the assumption that PSR J0529-6652 is exhibiting core emission, as suggested by its simple profile morphology, narrow profile width, and previously measured profile polarization characteristics.
\end{abstract}

Key words: pulsars: individual (PSR J0529-6652) - radiation mechanisms: non-thermal

Online-only material: color figure

\section{INTRODUCTION}

From the time of their discovery more than 40 years ago, the detailed mechanism by which pulsars generate radio emission has remained elusive. Models have been proposed over that time to explain pulsar emission, and in principle these models can be tested with pulsar observations. One relatively recent emission model, proposed by Gil \& Sendyk (2000), is an extension of the polar cap spark model of Ruderman \& Sutherland (1975) in which bursts of plasma ("sparks") are produced at the polar cap. In this model, instabilities in the plasma traveling along the magnetic field lines induce radio emission. The model postulates a densely packed region of these sparks, with a characteristic size and separation $h$. One can determine a characteristic number of sparks $a$ (in one dimension) across the polar cap according to

$$
a=r_{p} / h,
$$

where $r_{p}$ is the polar cap radius. In the Gil \& Sendyk (2000) model, $a$ is a "complexity parameter" that can also be determined observationally by

$$
a=5\left(\dot{P} / 10^{-15}\right)^{2 / 7}(P / 1 \mathrm{~s})^{-9 / 14},
$$

where $P$ and $\dot{P}$ are the pulsar's spin period and period derivative, respectively. There are several other similar models having different scalings to determine the complexity parameter (see, e.g., Burke-Spolaor et al. 2012 for a brief summary and references to these models). Jenet \& Gil (2003) provide details on how such models might be tested by comparing a pulsar's observed intensity modulation with the variability predicted by the complexity parameter in the model. Although the Gil \& Sendyk (2000) model does not make a distinction between core and conal emission from the pulsar (Rankin 1983), a model test is most useful using pulsars with core emission, since observationally it is the most straightforward probe of the polar cap region, it is not affected by viewing geometry effects from an off-center cut through the emission region, and it is less likely to exhibit cone-based pulse drifting effects which would increase the observed modulation (Weltevrede et al. 2006a).

One measure of a pulsar's intensity variability that can be employed in such tests is the phase-resolved modulation index, $m(\phi)$. Here $\phi$ is the longitude of the pulse phase and $m(\phi)$ is the ratio of the standard deviation $\sigma(\phi)$ of the observed pulse intensities to the mean pulse intensity averaged over many adjacent pulses, $\langle I(\phi)\rangle$ (Jenet \& Gil 2003; Weltevrede et al. 2006a):

$$
m(\phi)=\frac{\sigma(\phi)}{\langle I(\phi)\rangle}=\frac{\sqrt{\left\langle I(\phi)^{2}\right\rangle-\langle I(\phi)\rangle^{2}}}{\langle I(\phi)\rangle} .
$$

As outlined in Jenet \& Gil (2003), one would expect an anti-correlation between $a$ and $m$ owing to the likelihood that overlapping sparks in a complex emission region (large $a$ ) would wash out and tend to reduce the degree of modulation, creating more steady emission (smaller $m$ ). The phase information is important to retain since the measured modulation index near the edges of a profile peak usually increases relative to the center, in some cases because conal emission may be present. The center of the peak of the pulse profile therefore offers the best measurement of the properties of the core emission. The largest signal is also present at the profile peak, so the most precise constraint on $m(\phi)$ can generally be made at that pulse phase. 
The modulation index has previously been measured for a number of radio pulsars. Weltevrede et al. (2006a) observed 187 pulsars at $1400 \mathrm{MHz}$ to investigate the modulation and sub-pulse drifting properties of the sample, and they produced modulation index measurements for 175 of them. More recently, Burke-Spolaor et al. (2012) reported measurements for a sample of 103 pulsars from the HTRU $1400 \mathrm{MHz}$ survey (Keith et al. 2010). Jenet \& Gil (2003) analyzed 2 pulsars observed near $1400 \mathrm{MHz}$ and re-analyzed another 10 that were previously observed at $430 \mathrm{MHz}$ (Jenet et al. 2001; Weisberg et al. 1986). The Jenet \& Gil (2003) sample includes a subset of 28 pulsars analyzed by Weisberg et al. (1986) at $430 \mathrm{MHz}$. Jenet \& Gil (2004) also measured $m$ for PSR B1937+21 at $1400 \mathrm{MHz}$. Other measurements of $m$ (but not phase resolved) were reported in Table 4 of Weltevrede et al. (2011) for members of several neutron star classes having different types of emission. These objects were the Crab pulsar-a classical giant pulse emitter (Staelin \& Reifenstein 1968; Argyle \& Gower 1972), the radio-emitting magnetar XTE J1810-197 (Camilo et al. 2006), the rotating radio transient (RRAT) J1819-1458 (McLaughlin et al. 2006), and the possible RRAT-link pulsar PSR B0656+14 (Weltevrede et al. 2006b). The modulation indices reported for three of these four sources were for frequencies near $1400 \mathrm{MHz}$; PSR B0656+14 was measured at $327 \mathrm{MHz}$.

Generally, $m$ is observed to be small, with typical values between about 0.5 and 1 (Weltevrede et al. 2006a, 2011; BurkeSpolaor et al. 2012). The histogram of measured modulation indices shown in Figure 8 of Weltevrede et al. (2006a) shows no measured $m$ with a value above 2, and only 2 of the 103 measurements of Burke-Spolaor et al. (2012) have $m>2$. As seen in Figure 1 of Jenet \& Gil (2003) and Figure 10 of Weltevrede et al. (2006a), the anti-correlation between $m$ and $a$ in the observed sample of pulsars is weak. This is also seen in the Burke-Spolaor et al. (2012) sample. One reason may be the inclusion of pulsars with conal emission in these larger samples; the modulation index and complexity parameter are not predicted to be as strongly correlated for conal emitters as they are for pulsars with core emission (Jenet \& Gil 2003; Burke-Spolaor et al. 2012). Another difficulty in establishing correlations is that the range of $m$ measured in the observed data set is small. One well-known exception to this is the Crab pulsar. The Crab's modulation index is due to its well-studied giant pulses (Staelin \& Reifenstein 1968; Argyle \& Gower 1972; Lundgren et al. 1995; Weltevrede et al. 2006a; Karuppusamy et al. 2010). However, this pulsar is somewhat atypical since it is young and very energetic and is also a well-known X-ray emitter. In addition, the Crab pulsar is difficult to classify as a core or conal emitter (Rankin 1990). The Crab may have conal emission and other complicating geometric profile features that could produce a larger measured modulation index, making its use for a simple test of the Gil \& Sendyk (2000) model difficult.

However, PSR J0529-6652 is one pulsar that could be used for a consistency check of the Gil \& Sendyk (2000) model. This is a $976 \mathrm{~ms}$ pulsar that was first discovered in a $600 \mathrm{MHz}$ pulsar survey of the Large Magellanic Cloud (LMC), and it was the first extragalactic pulsar discovered (McCulloch et al. 1983). After its discovery, the pulsar was studied at frequencies near $600 \mathrm{MHz}$ by McConnell et al. (1991) and Costa et al. (1991), and timing results for the pulsar were subsequently published by Crawford et al. (2001).

PSR J0529-6652 was redetected as a known pulsar in a more recent $1400 \mathrm{MHz}$ Parkes survey of the Magellanic Clouds (MCs) for pulsars (Crawford et al. 2001; Manchester et al. 2006).
In these redetections, it was evident that the pulsar was variable on a timescale of minutes or less and showed possible nulling behavior, unlike any of the other MC radio pulsars detected. PSR J0529-6652 also had clearly detectable single radio pulses in these observations, making this pulsar phenomenologically unlike every other extragalactic radio pulsar yet discovered. ${ }^{3}$ PSR J0529-6652 is very luminous at $1400 \mathrm{MHz}$, ranking in the top $2 \%$ of the known radio pulsar population in the ATNF pulsar catalog (Manchester et al. 2005). However, apart from its luminosity and apparent nulling behavior, the pulsar is unremarkable, with a period and period derivative that are typical of the unrecycled radio pulsar population.

Previous measurements of PSR J0529-6652 taken with Parkes at $600 \mathrm{MHz}$ by Costa et al. (1991) showed that the pulsar has a simple, single-peaked morphology, with little pulse broadening. The width of the measured profile is $\sim 3 \%$ of the period at $600 \mathrm{MHz}$ (Costa et al. 1991). The integrated pulse profile of PSR J0529-6652 at $1400 \mathrm{MHz}$ is also singlepeaked and uncomplicated (see our analysis below), and its width at both frequencies is consistent with the empirical relation for the pulse width at $1 \mathrm{GHz}$ of $W=2.45 P^{-1 / 2} / \sin \alpha$ presented by Rankin (1990) for core emitters, if a reasonable magnetic inclination angle is assumed $\left(\alpha \sim 15^{\circ}\right)$. These features are consistent with a core emission interpretation for PSR J0529-6652. A polarization profile at $600 \mathrm{MHz}$ from Costa et al. (1991) shows significant linear polarization $(\sim 25 \%)$, with weaker circular polarization that changes sign symmetrically near the profile peak. This sign reversal also supports the interpretation of PSR J0529-6652 as a single core emitter (Rankin 1990). In addition, the majority of known pulsars ( $\sim 70 \%$; Rankin 1990) that have been classified have core components. On this basis, we conclude that PSR J0529-6652 is likely to be exhibiting core emission, making it useful for a test of the Gil \& Sendyk (2000) model.

\section{OBSERVATIONS AND ANALYSIS}

In order to investigate the pulse variability characteristics of PSR J0529-6652, we observed the pulsar with the Parkes $64 \mathrm{~m}$ radio telescope in two separate observations of length 5.0 and $3.8 \mathrm{hr}$ in 2008 February. Both observations were conducted at a center frequency of $1390 \mathrm{MHz}$ using the center beam of the multibeam receiver and analog filterbank system (StaveleySmith et al. 1996; Manchester et al. 2001). A bandwidth of $256 \mathrm{MHz}$ was split into $512 \times 0.5 \mathrm{MHz}$ channels, and each channel was 1 bit digitized and sampled every $500 \mu$ s (see Table 1). The observing setup was identical to the one used in a radio pulsar search of the X-ray binary XTE J0103-728 in the Small Magellanic Cloud (Crawford et al. 2009), which was part of the same observing campaign. Radio frequency interference (RFI) can be a significant problem at $1400 \mathrm{MHz}$ at Parkes, and much of the data for PSR J0529-6652 was at least partially corrupted by RFI. We selected a portion of the first observation that was clean of RFI for the analysis. This subset consisted of 4195.5 s of integration, corresponding to 4299 complete pulses.

We performed the following operations on PSR J0529-6652 as well as on three bright test pulsars, PSRs J0437-4715, $\mathrm{J} 0536-7543$, and $\mathrm{J} 1359-6038$, in order to test our processing

\footnotetext{
3 The LMC pulsar PSR B0540-69 also has detectable single radio pulses (Johnston \& Romani 2003), but it is not detectable as a periodic source at $1400 \mathrm{MHz}$. It is also a young, rapidly rotating X-ray emitter (Seward et al. 1984), making it different from PSR J0529-6652 in this respect as well.
} 
Table 1

Properties, Observing Parameters, and Measured Quantities for Four Pulsars

\begin{tabular}{|c|c|c|c|c|}
\hline Pulsar & J0529-6652 & J0437-4715 & J0536-7543 & $\mathrm{J} 1359-6038$ \\
\hline Topocentric period, $P(\mathrm{~s})^{\mathrm{a}}$ & 0.97573571876 & 0.00575786 & 1.24585559629 & 0.12751295 \\
\hline Dispersion measure, DM $\left(\mathrm{pc} \mathrm{cm}^{-3}\right)^{\mathrm{a}}$ & 103.2 & 2.6 & 17.5 & 293.7 \\
\hline Number of consecutive pulses used, $N$ & 4299 & 20839 & 240 & 1943 \\
\hline Number of profile bins & 128 & 128 & 128 & 128 \\
\hline Center observing frequency $(\mathrm{MHz})$ & 1390 & 1390 & 1390 & 1374 \\
\hline Sampling time $(\mu \mathrm{s})$ & 500 & 80 & 250 & 500 \\
\hline Scintillation bandwidth, $\delta v(\mathrm{MHz})^{\mathrm{b}}$ & 1.51 & 318 & 22 & $\sim 0$ \\
\hline Modulation index for the ISM, $m_{\mathrm{ISM}}{ }^{\mathrm{c}}$ & $0.20 \pm 0.03$ & $0.95 \pm 0.02$ & $0.61 \pm 0.07$ & $\sim 0$ \\
\hline Measured modulation index, $m^{\mathrm{d}}$ & $4.15 \pm 0.29$ & $1.00 \pm 0.02$ & $1.40 \pm 0.22$ & $0.33 \pm 0.03$ \\
\hline Intrinsic modulation index, $m_{i}{ }^{\mathrm{e}}$ & $4.07 \pm 0.29$ & $0.23 \pm 0.02$ & $1.08 \pm 0.22$ & $0.33 \pm 0.03$ \\
\hline Measured nulling fraction $(\mathrm{NF})^{\mathrm{f}}$ & $83.3 \% \pm 1.5 \%$ & $0.0 \% \pm 0.7 \%$ & $32.5 \% \pm 6.5 \%$ & $0.1 \% \pm 2.3 \%$ \\
\hline
\end{tabular}

Notes. All observations were taken with the center beam of the multibeam receiver and $512 \times 0.5 \mathrm{MHz}$ channel analog filterbank system at the Parkes telescope, except for PSR J1359-6038, where $96 \times 3 \mathrm{MHz}$ channels were used (Manchester et al. 2001).

${ }^{a}$ Obtained or derived from the ATNF pulsar catalog (Manchester et al. 2005).

b Estimated from the NE2001 Galactic electron model of Cordes \& Lazio (2002).

${ }^{\mathrm{c}}$ Estimated contribution to the modulation from propagation through the Galactic ISM.

${ }^{\mathrm{d}}$ Measured from the normalized pulse stack. The lowest value of $m(\phi)$ among the on-pulse bins was chosen (see Jenet \& Gil 2003 and the text for justification).

e Obtained after correction for the estimated Galactic ISM contribution.

f The uncertainty in NF was determined by $N^{-1 / 2}$, where $N$ is the number of pulses used.

algorithms. ${ }^{4}$ We dedispersed the raw channelized data for each pulsar using the catalog dispersion measure (DM) from the ATNF pulsar catalog (Manchester et al. 2005) ${ }^{5}$ to produce a dedispersed time series. We then created a pulse stack (e.g., Weltevrede et al. 2006a; Burke-Spolaor et al. 2012) by folding the dedispersed time series modulo the topocentric pulse period and stacking the resulting consecutive pulses. This produced a two-dimensional array consisting of pulse number versus pulse phase bin. In each case, 128 pulse phase bins were used for the pulse stack. We determined the mean pulse profile by summing the pulses in the pulse stack while preserving pulse phase. All elements in the pulse stack were then subsequently adjusted (normalized) so that the calculated mean intensity had zero mean for the off-pulse bins and unity peak value. The mean intensity and mean intensity squared were then recomputed for each bin using this normalized pulse stack. Figure 1 shows the normalized pulse stack for J0529-6652 and the three test pulsars for comparison, and Figure 2 shows the mean intensity profile for PSR J0529-6652. As is the case at $600 \mathrm{MHz}$, the mean pulse profile for PSR J0529-6652 at $1390 \mathrm{MHz}$ has a simple morphology and narrow width, with a duty cycle of 3-4 bins (corresponding to $\sim 25 \mathrm{~ms}$, or $\sim 3 \%$ of the pulse period). This similarity to the width measured by Costa et al. (1991) at $600 \mathrm{MHz}$ indicates that the pulse does not experience significant broadening at lower frequencies and remains narrow across a range of frequencies. The phaseresolved modulation index was measured for each profile phase bin using the normalized pulse stack according to Equation (3). An uncertainty in each modulation index value was computed using the rms values of the intensity and intensity squared within each bin and the number of points that were summed

\footnotetext{
4 All three test pulsars were observed with the same Parkes observing system as PSR J0529-6652 (but at different epochs). In the case of PSR J1359-6038, a slightly different center frequency $(1374 \mathrm{MHz})$ and filterbank system (consisting of $96 \times 3 \mathrm{MHz}$ channels) were used (Manchester et al. 2001; see Table 1).

5 http://www.atnf.csiro.au/research/pulsar/psrcat/
}

within each bin. The modulation index values were corrected to account for contributions from the interstellar medium (ISM; this is discussed in Section 3.2), and the resulting phase-resolved modulation index for PSR J0529-6652 is plotted and discussed later in the paper.

Nulling fractions (NFs) were also computed for PSR J0529-6652 and the three test pulsars using on and off-pulse intensity histograms that were created from the pulse stacks, as outlined by Wang et al. (2007). Prior to this, however, we tested our procedure by recomputing the NF for three known nulling pulsars measured at Parkes by Wang et al. (2007) at a center frequency of $1518 \mathrm{MHz}$. These pulsars were PSRs J1049-5833, $\mathrm{J} 1502-5653$, and J1525-5417 (see their Table 1). We compared our NF values as a check for consistency. Two of the archival Parkes observations that we used for this comparison were also taken at $1518 \mathrm{MHz}$, while the third (PSR J1049-5833) had a center frequency of $1318 \mathrm{MHz}$. The uncertainties in our measured NFs were estimated using $N^{-1 / 2}$, where $N$ is the number of subintegrations (pulses) used. This is a slightly more conservative estimate than that used by Wang et al. (2007), where the number of null subintegrations $n_{p}$ and total number of subintegrations $N$ were used in the expression $\sqrt{n_{p}} / N$. In all three test cases we obtained values that were consistent with the Wang et al. (2007) values considering our respective uncertainties and that different data sets were used. This NF comparison is presented in Table 2. As mentioned below, the NF measured for PSR J0529-6652 may be influenced by the much larger distance to the pulsar than to the test pulsars, possibly making many of the pulses from PSR J0529-6652 unseen.

\section{RESULTS AND DISCUSSION}

\subsection{Giant Pulses from PSR J0529-6652 and the Nulling Fraction}

Individual pulses from PSR J0529-6652 were clearly detected in the $4195.5 \mathrm{~s}$ subset of the observation used in the analysis (Figure 3). Three of these pulses, shown in the lower 

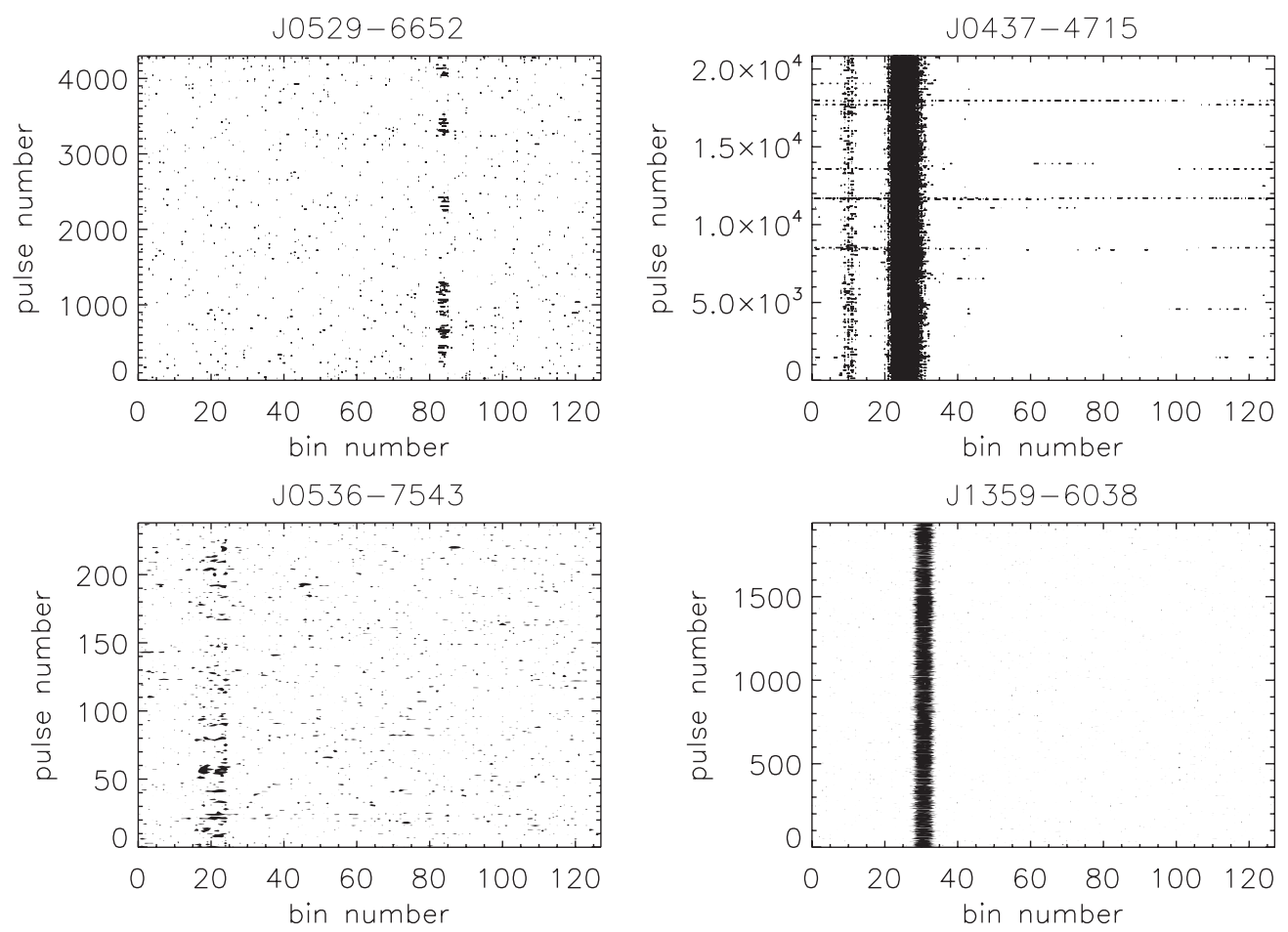

Figure 1. Pulse stacks for PSR J0529-6652 and three bright test pulsars (PSRs J0437-4715, J0536-7543, and J1359-6038). Each pulse stack has 128 pulse phase bins (horizontal axis) but a different number of consecutive pulses (vertical axis). Table 1 lists the observing parameters and ATNF catalog properties for the four pulsars. The contrast in each plot has been adjusted to best illuminate any variability in the pulses. All observations were taken near $1400 \mathrm{MHz}$, and the data are largely free of RFI. The variability is clearly evident in the PSR J0529-6652 pulse stack, consistent with its large measured NF (Table 1).

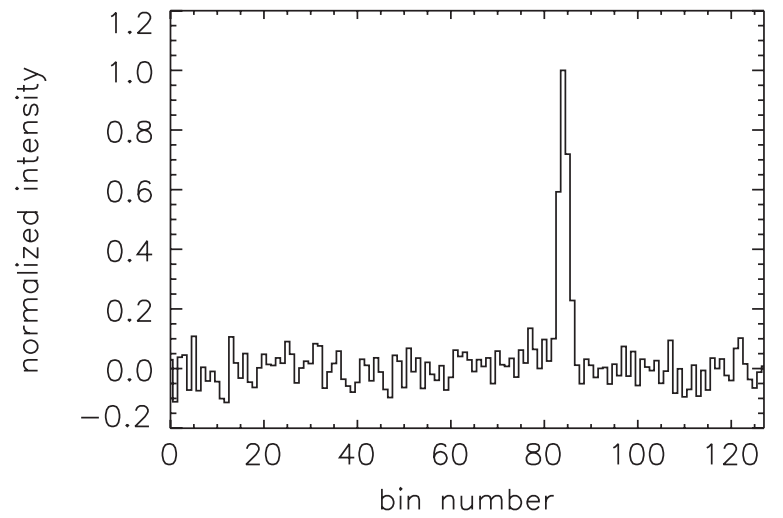

Figure 2. Normalized mean intensity profile for PSR J0529-6652 from the addition of 4299 consecutive pulses from a $1390 \mathrm{MHz}$ Parkes observation. There are 128 phase bins in the profile, and the profile has unity peak value and an off-pulse mean of zero. The mean pulse profile is narrow and uncomplicated, with a width of 3-4 bins ( $\sim 25 \mathrm{~ms}$, or $\sim 3 \%$ of the pulse period), and has no obvious additional or outlying components. These features, plus the polarization characteristics measured at $600 \mathrm{MHz}$ by Costa et al. (1991), suggest that PSR J0529-6652 is likely to be exhibiting core emission.

part of Figure 3, were constructed using the dedispersed data. In these three plots, the intensity (shown on the vertical axis) was calculated by equating the rms of the dedispersed time series to the noise level as determined by the radiometer equation (see, e.g., Table 1 of Burke-Spolaor et al. 2011). The pulse flux density was calculated from the rms and the pulse signal-tonoise ratio $(\mathrm{S} / \mathrm{N})$, which ranged from 8 to 11 for these three pulses. The resulting flux densities ranged from $S \sim 420$ to $575 \mathrm{mJy}$ at $1400 \mathrm{MHz}$. Using the definition of the pseudoluminosity (e.g., Lorimer \& Kramer 2004), $L=S d^{2}$, and assuming an LMC distance of $d \sim 50 \mathrm{kpc}$ (Koerwer 2009), this corre-
Table 2

Nulling Fractions for Three Known Nulling Pulsars

\begin{tabular}{lccr}
\hline \hline Pulsar & Wang et al. (2007) & This Work & $N^{\mathrm{b}}$ \\
\hline J1049-5833 & $47 \% \pm 3 \%$ & $33 \% \pm 35 \%$ & 8 \\
J1502-5653 & $93 \% \pm 4 \%$ & $70 \% \pm 9 \%$ & 120 \\
J1525-5417 & $16 \% \pm 5 \%$ & $26 \% \pm 5 \%$ & 361 \\
\hline
\end{tabular}

Notes. For all three pulsars, different sets of Parkes data were used for our measurements than were used by Wang et al. (2007). All of the data used in both sets of measurements had a center frequency of $1518 \mathrm{MHz}$, except for our measurement of PSR J1049-5833, which had a center frequency of $1318 \mathrm{MHz}$. ${ }^{\text {a }}$ Values from Table 1 of Wang et al. (2007).

${ }^{b}$ Number of subintegrations used in our NF measurements. The uncertainty in the NF was determined by $N^{-1 / 2}$.

sponds to $1400 \mathrm{MHz}$ peak luminosities of between 1050 and $1440 \mathrm{Jy} \mathrm{kpc}^{2}$. All three pulses have widths of $\sim 20 \mathrm{~ms}$, corresponding to about 40 time samples, which is comparable to the width of the integrated pulse profile shown in Figure 2. This is much larger than either the dispersion smearing within the frequency channels $(\sim 0.15 \mathrm{~ms})$ or the expected pulse scattering time $\left(\sim 10^{-4} \mathrm{~ms}\right)$ from Galactic plasma at $1400 \mathrm{MHz}$, as estimated from the NE2001 electron model (Cordes \& Lazio 2002). This indicates that the pulses are not giant micropulses or very narrow single pulses like those seen for the Crab and Vela pulsars and for PSR B1937+21 (Cognard et al. 1996; Johnston et al. 2001; Johnston \& Romani 2004). The three pulses shown also occur at the same pulse phase as the peak of the integrated profile.

A comparison of pulse intensities in the sample to the average pulse intensity indicates that some pulses have intensities $\gtrsim 20$ times the average. This can be seen in Figure 4, which shows the pulse intensity histogram. These pulses may be 

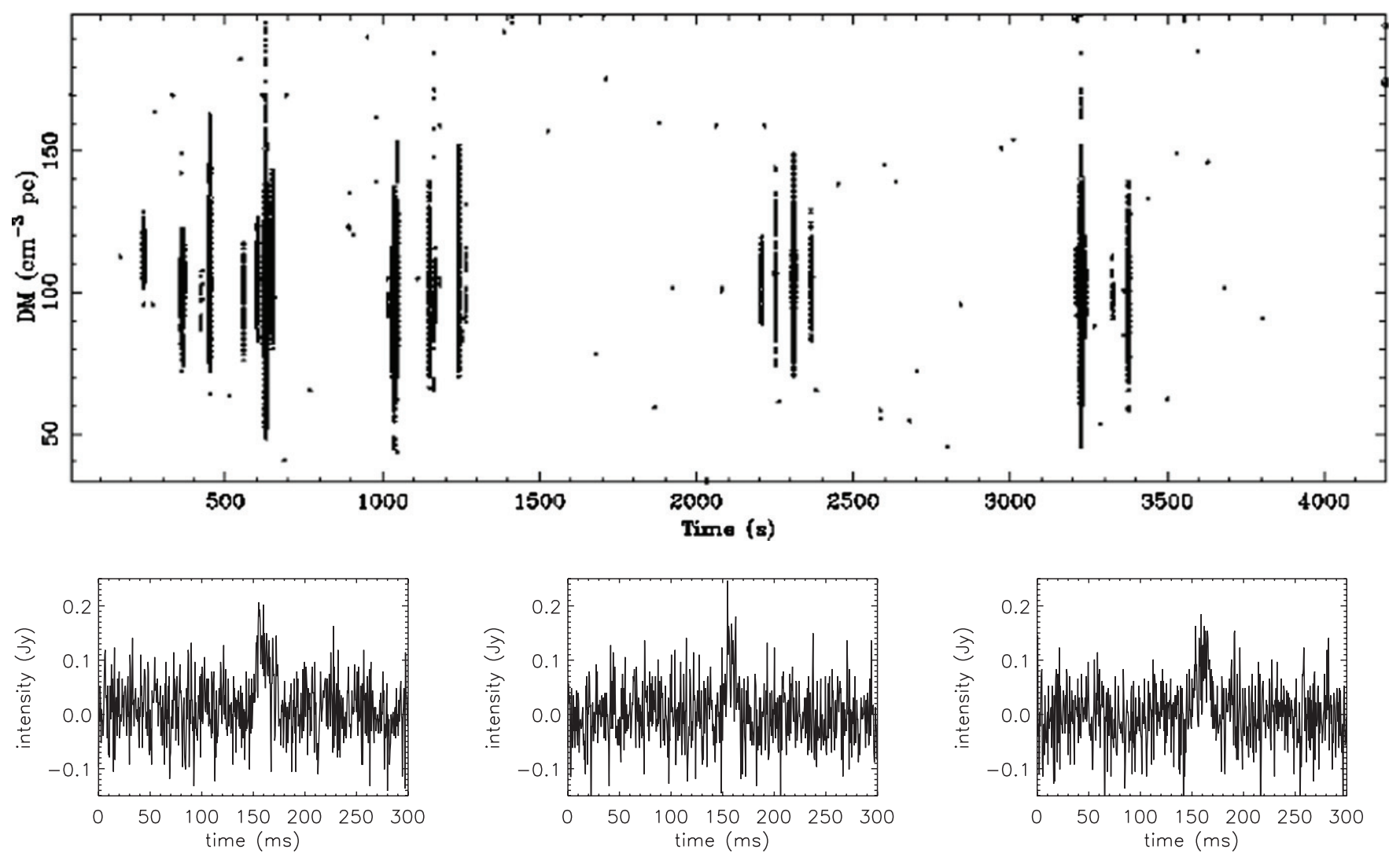

Figure 3. Single pulse detections of PSR J0529-6652 at $1390 \mathrm{MHz}$ from $4195.5 \mathrm{~s}$ of integration time, or 4299 pulses. The data here are the same data that were used to make the pulse stack and to measure the modulation index and NF (see Table 1). The top frame shows pulse strength as a function of both DM and time, with pulse events with $\mathrm{S} / \mathrm{N}>5.5$ shown. As expected, the pulses occur most strongly near the pulsar's DM of $\sim 100 \mathrm{pc} \mathrm{cm}^{-3}$. The lower frames show three detectable pulses from this observation plotted as intensity vs. time after dedispersion was applied. In all three cases, the pulses have a width of $\sim 20 \mathrm{~ms}(\sim 2 \%$ of the pulse period), or roughly 40 samples. This is comparable to the width of the integrated pulse profile (see Figures 2 and 5). Since the pulses are not dispersion or scatter broadened, this suggests that we are seeing the intrinsic widths and that the pulses are not giant micropulses. These three pulses also occur at the same pulse phase as the integrated profile. PSR J0529-6652 is the second pulsar in the LMC (after PSR B0540-69) known to emit detectable single radio pulses. The pulses shown here illustrate the high degree of amplitude variability for the pulsar, which is confirmed by the large measured modulation index and NF.

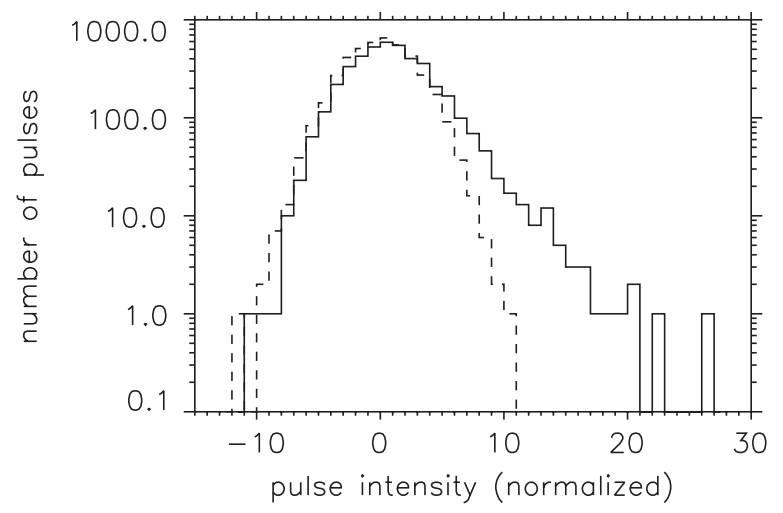

Figure 4. Histogram of on-pulse intensities for PSR J0529-6652 from the subset of 4299 pulses used in the analysis (solid line). The corresponding offpulse intensities calculated using the same number of off-pulse phase bins for each pulse are also shown (dashed line). Both histograms have been normalized to the mean on-pulse intensity value. These histograms were used in the calculation of the NF for PSR J0529-6652. There is an excess of pulses with large amplitudes, extending well beyond the noise limit, indicating that PSR J0529-6652 is a giant pulse emitter. We are unable to distinguish between a power-law and lognormal distribution for the giant pulses owing to the relatively small number of pulses detected.

considered giant pulses, which are generally defined as having amplitudes $>10$ times the average (Johnston et al. 2001; Knight 2006; Karuppusamy et al. 2011). However, it is not clear from the small number of high-intensity pulses shown in the histogram in Figure 4 whether the giant pulses follow a powerlaw distribution in intensity, as expected for classical giant pulses (e.g., Argyle \& Gower 1972), or possibly a lognormal distribution, as in the case of PSR B0656+14 (Weltevrede et al. 2006b; see discussion below). A fit to the data is unable to distinguish between these two cases. Future observations should provide better statistics and should be able to resolve this question. There is also no evidence of any association with a high-energy emission mechanism for PSR J0529-6652, which is thought to be a feature of classical giant pulses (Johnston \& Romani 2004). We checked this with a high-resolution XMM-Newton map of the region (M. Filipović 2012, private communication; see also Bozzetto et al. 2012) and by folding Fermi Large Area Telescope data using the pulsar's ephemeris to look for gamma-ray pulsations. No emission was detected from the pulsar in either case.

Another test for the presence of giant pulses is an $R$-parameter, defined by Johnston et al. (2001) and described by BurkeSpolaor et al. (2012). A value $R$ is determined for each profile bin by taking the difference between the maximum value and the mean value of the samples for that bin, which is then divided by the rms of the values for that bin. A subsequent comparison of the $R$-value for each bin with the rms of the $R$-values for the off-pulse bins can be used as a threshold test for the presence of sparse modulated emission, such as giant pulses. Following Burke-Spolaor et al. (2012), we use a significance threshold defined as $R$ minus the off-pulse mean of the $R$-values, divided 
by the standard deviation of $R$ in the off-pulse window. If this quantity is greater than 4 for any phase bin, then giant pulses are considered to be present. For three of the four on-pulse bins for PSR J0529-6652, $R$ was larger than 6 and nowhere else in the profile was it above 4 . This supports our conclusion that giant pulses from PSR J0529-6652 are present and that they do not significantly lead or trail the main pulse.

As seen in Figure 3, there are at least 15 distinct pulses that are visible by eye that were also detected in the single pulse search detection algorithm (Cordes \& McLaughlin 2003). This corresponds to one detectable pulse every $\sim 4.5$ minutes on average, though it is evident that the pulses are not evenly spaced. For comparison, we consider two young, canonical giant radio pulse emitters: the Crab pulsar and PSR B0540-69. Crawford et al. (2005) used the same Parkes observing system as used here (but with a different sampling rate) to observe the X-ray pulsar PSR J0537-6910 in the LMC at $1400 \mathrm{MHz}$ (Marshall et al. 1998). They calculated that if the Crab pulsar were located in the LMC, one pulse would be expected to be detectable with this system every $\sim 20$ minutes. In the case of PSR B0540-69, which is in the LMC, one giant pulse should be detectable every $\sim 30$ minutes. ${ }^{6}$ Thus, the rate of detectable pulses per unit time from PSR J0529-6652 with this system is almost an order of magnitude greater than the expected rates for either the Crab pulsar (if it were in the LMC) or PSR B0540-69. Moreover, the spin period of PSR J0529-6652 (976 ms) is more than an order of magnitude larger than either the Crab pulsar (33 ms) or PSR B0540-69 (50 ms). Therefore, the likelihood that any one pulse from PSR J0529-6652 would be detectable as a giant pulse with this system (or, alternatively, the fraction of emitted pulses detectable as single pulses with this system) is about two orders of magnitude greater than for either the Crab (if it were in the LMC) or PSR B0540-69. Unlike these two pulsars, however, PSR J0529-6652 is physically unremarkable, making this feature of its emission quite unusual and unexpected.

The pulse characteristics from PSR J0529-6652 appear to be more similar to those from PSR B0656+14 (Weltevrede et al. 2006b; Tao et al. 2012). The pulses from PSR B0656+14 follow a lognormal distribution rather than a power law (e.g., Figure 1 of Weltevrede et al. 2006b), which might also be the case for PSR J0529-6652 (see Figure 4), they are not associated with any high-energy emission, and they are not narrow micropulses. PSR B0656+14, like PSR J0529-6652, also does not have a large magnetic field at the light-cylinder radius, which is a feature of known classical giant pulse emitters (Cognard et al. 1996). These features make both PSR J0529-6652 and PSR B0656+14 different from classical giant pulse emitters. As discussed below, the large modulation indices measured for PSRs B0656+14 and J0529-6652 and the proximity of both pulsars to the bulk of the unrecycled pulsar population on the period/period derivative diagram also suggest a similarity between these two sources.

We measured NFs for PSR J0529-6652 and our three test pulsars. These values are presented in Table 1. Both PSRs J0437-4715 and J1359-6038 have essentially no nulling near $1400 \mathrm{MHz}(\mathrm{NF} \sim 0)$. The NF for PSR J0529-6652 at $1390 \mathrm{MHz}$ is $83.3 \% \pm 1.5 \%$, indicating that either radio emission is not present for the majority of the pulse periods or that most pulses

\footnotetext{
6 This expected detection rate for PSR B0540-69 is somewhat larger than the actual detection of only one pulse in a recent $1.4 \mathrm{hr}$ test observation that had comparable sensitivity. This observation was taken as part of new multibeam pulsar survey of the LMC with Parkes.
}

are simply too weak to be distinguishable from noise. This latter point is an important possibility given the very large distance to PSR J0529-6652. In all four cases the measured NFs appear qualitatively consistent with the pulse stacks shown in Figure 1.

\subsection{The Modulation Index of PSR J0529-6652}

For the modulation index calculation, we first estimated the contribution to the modulation from the Galactic ISM for each of the pulsars, and we corrected the measured modulation indices for this to obtain the value intrinsic to each pulsar. As outlined in Jenet \& Gil (2003), the measured $(m)$, intrinsic $\left(m_{i}\right)$, and ISM-produced $\left(m_{\mathrm{ISM}}\right)$ modulation indices are related according to

$$
\left(m^{2}+1\right)=\left(m_{i}^{2}+1\right)\left(m_{\mathrm{ISM}}^{2}+1\right) .
$$

$m_{\text {ISM }}$ can be estimated using the relationship (Jenet et al. 2001; Jenet \& Gil 2003)

$$
m_{\mathrm{ISM}}=\frac{1}{(1+\eta B / \delta \nu)^{1 / 2}},
$$

where $B$ is the observing bandwidth, $\delta v$ is the characteristic ISM diffractive scintillation bandwidth, and $\eta$ is a coefficient ranging from 0.1 to 0.2 (Cordes et al. 1990). $\delta v$ was determined at $1400 \mathrm{MHz}$ for each pulsar using the NE2001 model of Cordes \& Lazio (2002) and the pulsar's catalog DM. For PSR J0529-6652, we used the maximum Galactic DM contribution along its line of sight according to the NE2001 model $\left(52 \mathrm{pc} \mathrm{cm}^{-3}\right)$. The uncertainty in $m_{\mathrm{ISM}}$ was calculated using a range of $\eta$ of $0.1-0.2$. Following the method of Jenet \& Gil (2003), the uncertainty in the intrinsic modulation index $m_{i}$ was determined by using the larger of the measurement uncertainties in $m$ and $m_{\text {ISM }}$. The results are presented in Table 1.

It should be noted that in the case of PSR J0529-6652, $m_{\text {ISM }}$ does not include any contribution from propagation through the LMC, where the pulsar resides. There is no model that we can use to estimate the LMC contribution to the modulation, but observations of other known pulsars in the MCs do not show any of the extreme variability and null-like behavior that is seen for PSR J0529-6652 (Crawford et al. 2001; Manchester et al. 2006). We conclude from this that the contribution to the modulation from the LMC itself is likely to be minimal and does not significantly affect our results.

After correcting for the estimated Galactic ISM contribution, we derived an intrinsic modulation index value for PSR J0529-6652 for each profile phase bin. Figure 5 shows the modulation indices for the on-pulse profile bins overlaid with the profile intensity. We selected the bin with the highest $\mathrm{S} / \mathrm{N}$ (the profile peak) for the analysis, which also had the most precise modulation index measurement. As stated in Jenet \& Gil (2003), this is the bin that is least likely to be affected by outlying conal emission components, which can introduce additional variability and can complicate the interpretation of the modulation index as a feature of the polar cap physics. This bin also had the lowest value of $m_{i}(\phi)$, as expected (see Jenet \& Gil 2003), and from this we derived an intrinsic modulation index of $4.07 \pm 0.29$ for PSR J0529-6652 (Table 1). The modulation indices measured for our three test pulsars using the same method are also presented in Table 1 . As expected, these values are all small $\left(m_{i} \lesssim 1\right)$ and are consistent with the range seen for most radio pulsars (see, e.g., Figure 6).

Figure 1 shows the pulse stack for PSR J0529-6652, which clearly shows its variability. As stated above, the LMC itself 


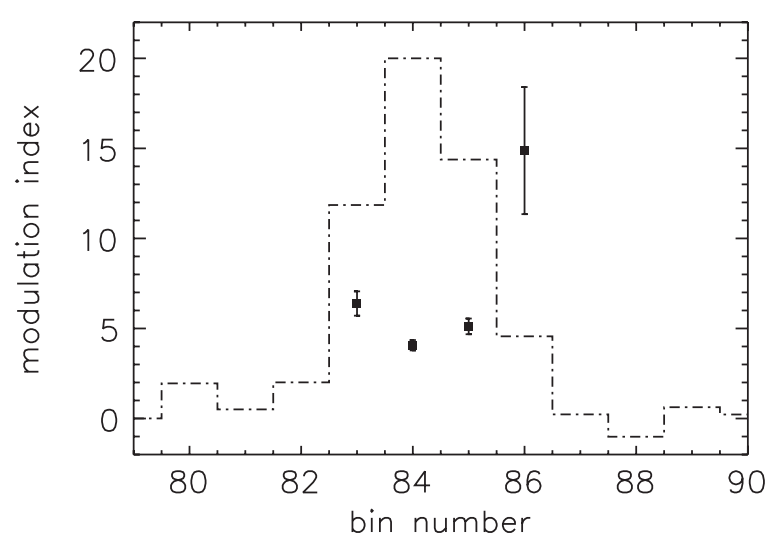

Figure 5. Phase-resolved intrinsic modulation index values measured for PSR J0529-6652 (squares) overlaid with corresponding mean intensity values (dash-dotted line). The intensity values have been arbitrarily scaled for display purposes. Only the pulse phase bins near the pulse peak are shown, and only the on-pulse bins have enough signal for reliable modulation index measurements. These modulation index values have already been corrected for the estimated contribution from fluctuations from the Galactic ISM. The minimum and most precise value of $m_{i}=4.07 \pm 0.29$ is seen at the profile peak, and this is the value used in our analysis (see, e.g., Jenet \& Gil 2003).

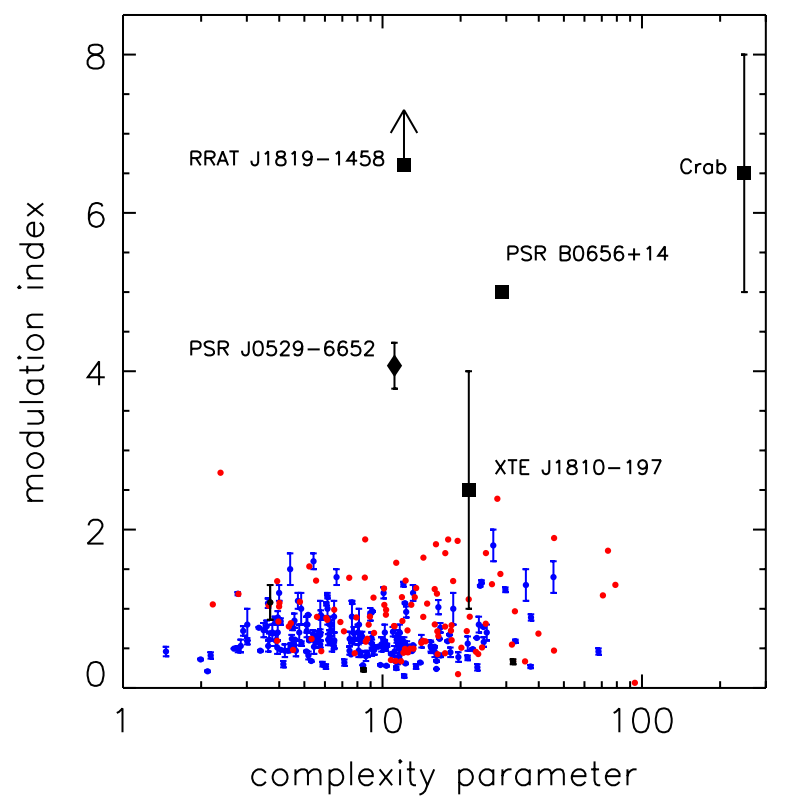

Figure 6. Intrinsic modulation index vs. complexity parameter determined from the Gil \& Sendyk (2000) model is shown for several samples of pulsars. All of the 174 pulsars measured by Weltevrede et al. (2006a, and presented in their Table 2 and Figure 10) are presented as small blue circles with error bars. Also plotted are the three measurements of our test pulsars (small black circles with errors). The 102 (out of 103) pulsars from Table 1 of Burke-Spolaor et al. (2012) with cataloged $\dot{P}$ values are plotted with red circles (no errors were reported). Our measurement for PSR J0529-6652 (large diamond) and the measurements presented by Weltevrede et al. (2011) in their Table 4 for four members of different, more variable neutron star classes are also shown (large squares). All data shown here were taken near $1400 \mathrm{MHz}$, except for PSR B0656+14, which was at $327 \mathrm{MHz}$. PSR J0529-6652 has physical parameters that are typical of the unrecycled radio pulsar population (see Figure 7), unlike the other labeled neutron stars (except possibly PSR B0656+14).

(A color version of this figure is available in the online journal.)

is likely not a major contributor to this variability. The diffraction scintillation timescale at $1400 \mathrm{MHz}$ for PSR J0529-6652 from Galactic plasma is $\sim 1500 \mathrm{~s}$ (Cordes \& Lazio 2002), significantly longer than the minute timescales (or less) that seem to be present qualitatively in the pulse stack. The scintillation bandwidth is also much smaller than the observing bandwidth

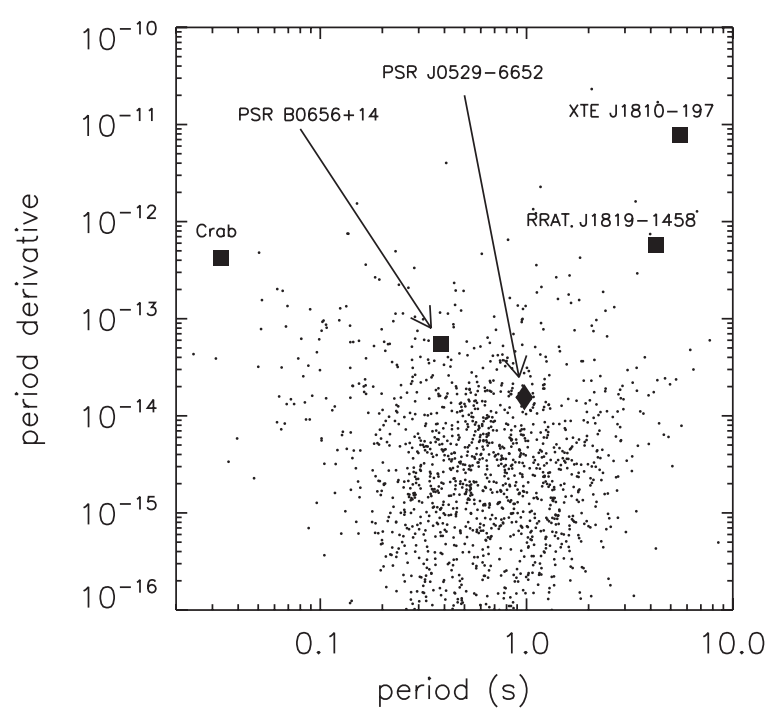

Figure 7. Period derivative vs. period for radio pulsars from the ATNF catalog (Manchester et al. 2005; small dots). Not all pulsars are shown (e.g., recycled millisecond pulsars are beyond the plot limits). Also shown are the four neutron stars with modulation index measurements presented in Table 4 of Weltevrede et al. (2011; squares). PSR J0529-6652 is shown as the large diamond. Compared to the other four labeled neutron stars, PSR J0529-6652 lies closer to the center of the unrecycled radio pulsar population and has spin characteristics that are more typical of this population.

(see Table 1). This suggests that the variability of the emission is intrinsic to the pulsar and is consistent with the large intrinsic modulation index measured.

Figure 6 shows the intrinsic modulation index versus the complexity parameter derived using the Gil \& Sendyk (2000) model for several samples of pulsars. One hundred and seventyfour pulsars measured by Weltevrede et al. (2006a) are plotted as small blue dots (see their Table 2 and Figure 10; note that their Crab measurement was excluded in their figure and is also not included here). In addition, 102 of the 103 pulsars measured by Burke-Spolaor et al. (2012) for which there was also a cataloged $\dot{P}$ are plotted as small red dots, but no error bars are given for these values. Our three test pulsars are plotted as small black dots with error bars, and our measurement for PSR J0529-6652 is indicated by the large diamond (see also Table 1). All of these measurements were taken near $1400 \mathrm{MHz}$. Although PSR J0529-6652 has a complexity parameter and physical characteristics that are typical of the rest of the sample (and the overall radio pulsar population in general; see Figure 7), it nevertheless has a modulation index that lies well above this sample and the sample of 12 pulsars shown in Figure 1 of Jenet $\&$ Gil (2003). Only two of the pulsars measured in any of these samples (apart from PSR J0529-6652) have $m>2$.

The large modulation index for PSR J0529-6652 is closer to the ranges and limits presented in Table 4 of Weltevrede et al. (2011) for four members of different neutron star classes (these are plotted in Figure 6 as large squares). These four sources are the Crab pulsar (see above), the radio magnetar XTE J1810-197, the RRAT J1819-1458, and the RRATlike pulsar PSR B0656+14. All of the measurements of these sources were taken near $1400 \mathrm{MHz}$, except for PSR B0656+14, which was taken at $327 \mathrm{MHz}$. Like PSR J0529-6652, they all have modulation indices that are significantly larger than the values that have been measured for typical radio pulsars. However, with the possible exception of PSR B0656+14, none lies near the center of the unrecycled radio pulsar population on the period/period derivative diagram (Figure 7). Thus, only 
PSR J0529-6652 (and possibly PSR B0656+14) are typical pulsars in this sense. In any case, the core-like characteristics of the emission from PSR J0529-6652 (suggested by its simple and narrow profile morphology and its symmetric sign change in circular polarization), plus its unremarkable spin and physical parameters (Crawford et al. 2001; see also Figure 6), suggest that this pulsar is more useful for a test of the correlation predicted by the Gil \& Sendyk (2000) emission model than these other neutron stars, all of which are physically unusual in different ways. We conclude that the large modulation index measured for PSR J0529-6652 is not consistent with the Gil \& Sendyk (2000) emission model, which predicts a small modulation index for a relatively large complexity parameter. We also note that the radio magnetar PSR J1622-4950 (Levin et al. 2010) has a relatively small measured modulation index $(m \sim 0.5$ measured by Burke-Spolaor et al. (2012) at $1.4 \mathrm{GHz}$, and $m \sim 1.7$ measured by Levin et al. (2012) at $3.1 \mathrm{GHz}$ ). This indicates that not all magnetars exhibit extreme radio modulation like XTE J1810-197, just as PSR J0529-6652 illustrates that not all typical radio pulsars have low modulation.

\section{CONCLUSIONS}

We have studied the variability of PSR J0529-6652, a luminous radio pulsar in the LMC, using observations taken at $1390 \mathrm{MHz}$ with the Parkes $64 \mathrm{~m}$ telescope. The pulsar emits detectable single radio pulses that can be classified as giant pulses, making this the second known pulsar in the LMC (after PSR B0540-69) to do so. These pulses are comparable in width to and occur at the same phase as the integrated profile peak, suggesting that they are not giant micropulses. The characteristics of the giant pulses appear to be closer to those seen from PSR B0656+14 than from classical giant pulse emitters, such as the Crab pulsar. The fraction of the pulses emitted by PSR J0529-6652 that are individually detectable as single pulses at this frequency is two orders of magnitude greater than either PSR B0540-69 or the Crab pulsar (if the latter were located in the LMC). PSR J0529-6652 also appears to exhibit nulling behavior, and we have measured an NF of $83.3 \% \pm 1.5 \%$ for the pulsar. Pulsed radio emission is either not present for the majority of pulse periods, or it is too weak in most cases to be individually detectable above the noise. Given the large distance to the pulsar, this is a possibility that must be considered. Our measured intrinsic modulation index for PSR J0529-6652 is $4.07 \pm 0.29$, which is significantly larger than the values previously measured for typical radio pulsars with similar spin and physical characteristics. It is comparable to the larger values presented by Weltevrede et al. (2011) for several members of other neutron star source classes that are known to be radio variable. Unlike these other sources, however, PSR J0529-6652 has spin and physical characteristics that are typical of the unrecycled radio pulsar population. These features make this pulsar useful as a test of the Gil \& Sendyk (2000) emission model, and the large modulation index measured for PSR J0529-6652 does not appear to be consistent with the model prediction. This conclusion depends to some degree on the assumption that PSR J0529-6652 is exhibiting core emission, as seems to be the case given its simple profile morphology, narrow profile width, and previously measured polarization characteristics at $600 \mathrm{MHz}$.

We thank M. Filipović for providing us with an X-ray image of the region containing PSR J0529-6652. The Parkes radio telescope is part of the Australia Telescope, which is funded by the Commonwealth of Australia for operation as a National Facility managed by CSIRO. This work was supported in part by grants from Research Corporation, the Mount Cuba Astronomical Foundation, the National Radio Astronomy Observatory, and the Hackman Scholarship program at Franklin and Marshall College.

\section{REFERENCES}

Argyle, E., \& Gower, J. F. R. 1972, ApJL, 175, 89

Bozzetto, L. M., Filipović, M. D., Crawford, E. J., et al. 2012, MNRAS, 420, 2588

Burke-Spolaor, S., Bailes, M., Ekers, R., Macquart, J.-P., \& Crawford, F. 2011, ApJ, 727, 18

Burke-Spolaor, S., Johnston, S., Bailes, M., et al. 2012, MNRAS, 423, 1351

Camilo, F., Ransom, S. M., Halpern, J. P., et al. 2006, Natur, 442, 892

Cognard, I., Shrauner, J. A., Taylor, J. H., \& Thorsett, S. E. 1996, ApJL, 457,81

Cordes, J. M., \& Lazio, T. J. W. 2002, arXiv:astro-ph/0207156

Cordes, J. M., \& McLaughlin, M. A. 2003, ApJ, 596, 1142

Cordes, J. M., Wolszczan, A., Dewey, R. J., Blaskiewicz, M., \& Stinebring, D. R. 1990, ApJ, 349, 245

Costa, M. E., McCulloch, P. M., \& Hamilton, P. A. 1991, MNRAS, 252, 13

Crawford, F., Kaspi, V. M., Manchester, R. N., et al. 2001, ApJ, 553, 367

Crawford, F., Lorimer, D. R., Devour, B. M., Takacs, B. P., \& Kondratiev, V. I 2009, ApJ, 696, 574

Crawford, F., McLaughlin, M., Johnston, S., Romani, R., \& Sorrelgreen, E. 2005, AdSpR, 35, 1181

Gil, J. A., \& Sendyk, M. 2000, ApJ, 541, 351

Jenet, F. A., Anderson, S. B., \& Prince, T. A. 2001, ApJ, 546, 394

Jenet, F. A., \& Gil, J. 2003, ApJL, 596, 215

Jenet, F. A., \& Gil, J. 2004, ApJL, 602, 89

Johnston, S., \& Romani, R. W. 2003, ApJL, 590, 95

Johnston, S., \& Romani, R. W. 2004, in IAU Symp. 218, Young Neutron Stars and Their Environments, ed. F. Camilo \& B. M. Gaensler (San Francisco, CA: ASP), 315

Johnston, S., van Straten, W., Kramer, M., \& Bailes, M. 2001, ApJL, 549,101

Karuppusamy, R., Stappers, B. W., \& Serylak, M. 2011, A\&A, 525, A55

Karuppusamy, R., Stappers, B. W., \& van Straten, W. 2010, A\&A, 515, A36

Keith, M. J., Jameson, A., van Straten, W., et al. 2010, MNRAS, 409, 619

Knight, H. S. 2006, ChJAS, 6, 41

Koerwer, J. F. 2009, AJ, 138, 1

Levin, L., Bailes, M., Bates, S., et al. 2010, ApJL, 721, 33

Levin, L., Bailes, M., Bates, S. D., et al. 2012, MNRAS, 422, 2489

Lorimer, D. R., \& Kramer, M. 2004, Handbook of Pulsar Astronomy (Cambridge: Cambridge Univ. Press)

Lundgren, S. C., Cordes, J. M., Ulmer, M., et al. 1995, ApJ, 453, 433

Manchester, R. N., Fan, G., Lyne, A. G., Kaspi, V. M., \& Crawford, F. 2006, ApJ, 649,235

Manchester, R. N., Hobbs, G. B., Teoh, A., \& Hobbs, M. 2005, AJ, 129, 1993

Manchester, R. N., Lyne, A. G., Camilo, F., et al. 2001, MNRAS, 328, 17

Marshall, F. E., Gotthelf, E. V., Zhang, W., Middleditch, J., \& Wang, Q. D. 1998, ApJL, 499, 179

McConnell, D., McCulloch, P. M., Hamilton, P. A., et al. 1991, MNRAS, 249,654

McCulloch, P. M., Hamilton, P. A., Ables, J. G., \& Hunt, A. J. 1983, Natur, 303, 307

McLaughlin, M. A., Lyne, A. G., Lorimer, D. R., et al. 2006, Natur, 439,817

Rankin, J. M. 1983, ApJ, 274, 333

Rankin, J. M. 1990, ApJ, 352, 247

Ruderman, M. A., \& Sutherland, P. G. 1975, ApJ, 196, 51

Seward, F. D., Harnden, F. R., \& Helfand, D. J. 1984, ApJL, 287, 19

Staelin, D. H., \& Reifenstein, E. C. 1968, Sci, 162, 1481

Staveley-Smith, L., Wilson, W. E., Bird, T. S., et al. 1996, PASA, 13, 243

Tao, G.-C., Esamdin, A., Hu, H.-D., et al. 2012, RAA, 12, 006

Wang, N., Manchester, R. N., \& Johnston, S. 2007, MNRAS, 377, 1383

Weisberg, J. M., Armstrong, B. K., Backus, P. R., et al. 1986, AJ, 92, 621

Weltevrede, P., Edwards, R. T., \& Stappers, B. W. 2006a, A\&A, 445, 243

Weltevrede, P., Johnston, S., \& Espinoza, C. M. 2011, MNRAS, 411, 1917

Weltevrede, P., Stappers, B. W., Rankin, J. M., \& Wright, G. A. E. 2006b, ApJL, 645,149 